\title{
Study on the Promotion of the Development of Animation Teaching Resources for Students' Psychological Education in the Information Environment
}

\author{
Chang Liu \\ Computer Engineering Department \\ Suzhou Vocational University \\ Suzhou, China \\ lchang@jssvc.edu.cn
}

\author{
Zhao-bin Liu \\ Computer Engineering Department \\ Suzhou Vocational University \\ Suzhou, China \\ Chang-liu2004@163.com
}

\begin{abstract}
According to the feasibility analysis of the application of animation resources in students' psychological health education, it is tried to play the special educational advantages of animation resources in this paper so as to combine the development of animation resources with the students' psychological health education. Two aspects have been mainly discussed: the construction of animation resources and the modular architecture of students' mental health resource platform. And it is tried to establish a new method that can effectively promote the students' mental health education in the information environment.
\end{abstract}

Keywords-animation; teaching resources; psychological education; Information Environment

\section{INTRODUCTION}

With the rapid development of animation industry around the world, the animation has become one of the consumption products, such as movie and TV, for social mass entertainment and it has been also considered as the popular medium to transmit the information. At the same time, the audience of animation has been extended from children to all ages, especially for the immature college students. The contents that are directly or indirectly conveyed by the animation will inevitably affect the psychological development of college students. These interesting entertaining pictures with strong visual appeal can cater to the acceptance habits and tastes of college students to a great extent. Therefore, a lot of students have become the animation fans. In this sense, the function of animation has not been limited to the entertainment for these students and the educational function has become evitable. Especially in the case that the majority of college students have become the animation audiences, how to combine the animation which is welcomed by most of the students with the students' psychological health education and how to develop a new mental health educational method in the information environment has undoubtedly played an important role in the smooth implementation of students' psychological health education and the comprehensive and personality development of students' mental quality.

\section{THE FEASIBILITY ANALYSIS ON THE PROMOTION OF ANIMATION TEACHING RESOURCES FOR STUDENTS' PSYCHOLOGICAL HEALTH EDUCATION}

A. The animation teaching resources can promote the enthusiasm of students to learn the psychological knowledge

As an art form, the fundamental charm of animation is that it can produce the aesthetic and emotional strength. The art psychology believes that the appreciation of animation is the excellent stimulus of people's pleasant experience; no matter the animated content is reproducibility or nonreproducibility, they all like it. For the mental health education of students, the most important thing in the process of learning is the happiness. There are a lot of contents in the traditional teaching. If these contents are presented by text, they will be "dull". However, these contents will be "rich" if they are presented by the animation, which can fully promote the learning enthusiasm of students and inject the new vitality into the teaching.

B. The animation teaching resources can expand the capacity of students to learn the psychological knowledge

The learning speed of students to watch animation video or images is higher than the that of reading the text. The learning pace can be obviously accelerated and the learning capacity can be greatly expanded through the concise, clear and fast video viewing method.

\section{The animation teaching resources can expand the range of psychological knowledge for students}

Currently, a lot of universities have their own campus network or the specialized mental health counseling sites where the prepared animation resources can be uploaded to. Afterwards, the students can easily obtain these resources for learning at any time or any places.

\section{The animation teaching resources can promote the development of students' overall thinking}

For this integrated thinking method, the thinking ways in oral transmission medium ages, print media ages and electronic media ages have been integrated into the animation video images. At the same time, the mind, the emotions, the attitudes, the abilities and some others have 
been also integrated into the animation video images. Then, the limitations of conventional thinking can be surpassed. It is the integrated thinking that has been beyond the analytical, linear and mechanical thinking.

\section{ADOPTING THE MODERN INFORMATION TECHNOLOGY} TOOLS TO DEVELOP THE ANIMATION TEACHING RESOURCES

"Animation" is essentially the combination and the abbreviation of cartoon and comics. The former refers to the static frame and the latter refers to the non-stop playing process constituted by a lot of static frames. Therefore, the development of animation teaching resources can be divided into the development of cartoon resources and the development of comic resources. What's more, the cartoon resources can be divided into the $2 \mathrm{D}$ and the $3 \mathrm{D}$ performance forms. For these methods, it is required to concern that how to combine the mental health knowledge into the design process of animation without greatly affecting the interestingness and the entertainment. According to the dependencies between the various elements in the animation production process, the entire resource development process should be refined and decomposed so as to obtain the production process of animation resources which are suited to the psychological health education of students.

\section{A. Needs-based positioning}

The needs-based positioning is the first component element in the development of animation resources, which includes the target positioning, the content positioning and the location positioning. The determination of animation teaching resources for the students' psychological health educational teaching content is an essential part in the design of animation teaching resources.

\section{B. Text script}

The first step of the production of any film is writing the scripts. The topics of scripts should be developed according to the topics of psychological health education. The scripts can be completed through inviting some psychological experts. The script determines the ideological levels of future animations, the depth of teaching significance, the expressive techniques of films and the styles, etc. Therefore, the idea of the scripts should be distinct and the structure should be clear.

\section{Shape-designing}

The shape-designing not only includes the styles of characters, but also contains the design of environment and instruments. This is a entirely creative process. In this step, it is required to consider the application field of mental health education and it should be close to the true life as far as possible. At the same time, it is also necessary to adopt some popular design factors which can be easily accepted by the college students. Therefore, it is required to use the authenticity to macroscopically grasp the styles and the effect of art design.

\section{Shooting script}

Based on the scripts and the design, the scripts should be drawn into the pictures. The function of these pictures is that they will become the design drawings of future animations, in which the screen, the lines, the sound effects and the time calculation should be also contained. Afterwards, the people who are involved in the animation production will understand fully at a glance and the contents of story will be clear.

\section{E. Animation production}

When all the preparatory work is completed, it will be required to select the tools to produce the animation teaching resources according to the actual situation. The $2 \mathrm{D}$ animation production software generally includes FLASH, ANIMO, RETASPRO and USANIMATION, etc. And the 3D animation production software generally contains 3DMAX, MAYA and LIGHTWAVE, etc. If it is required to produce the static frame, the graphics software such as PHOTOSHOP, PAINTER and CORELDRAW can be selected which will draw on computers through combining with the tablet. It is necessary to adopt the authoring software for animation production and integrate the psychological health teaching contents into them according to the previous requirements. Then, the animation teaching resources with strong interestingness, strong ornamental feature or strong interaction will be produced.

\section{F. Evaluation and revision}

During the production of animation resources, every aspect of previous work can be evaluated and revised at any time.

\section{G. Generation of works}

It is required to consider the format of animations during the production. The works will be ultimately published to the network for teachers and students. Therefore, they should be generated with the streaming media format which can be easily disseminated online. At the same time, they can be also generated with the screen size and format which are suitable for mobile watching. Then, these resources can be downloaded by the mobile users.

\section{MODUlE FUnCTIONS OF ANIMATION TEACHING RESOURCE PLATFORM}

In order to integrate the developed animation educational resources into the network, it is required to establish a teaching resource of health education sharing and exchanging platform which can realize the psychological health education of students through the campus application network. The students can quickly and easily access and browse the rich animation-based psychological teaching resourcesthrough this platform. What's more, the students can also conduct the online learning activities and various exchanging and sharing activities. Based on the excellent resource management capacity, the service capacity and the reproduction capacity, this platform has provided the solutions for realizing the informationize applications of educational animation product resources. The animation resource psychological teaching platform includes the following six modules: the psychological emotion, the interesting psychology, the interesting test, the case study, 
the psychological theater and the drawing psychology. Each module can be embedded into the animation teaching resources.

\section{A. Module of psychological emotion}

This module takes the psychological teaching contents such as the workplace psychology, the growth psychology and the interpersonal psychology as the materials and it takes the health cartoons, 2D or 3D animated short films as the performance forms. The psychological knowledge is mainly transmitted in the form of storytelling. The animation teaching method makes the language vivid, the explanation detailed, deep and thorough, which can help the students easily comprehend the difficulties of the psychological teaching knowledge. This module aims to cultivate the health psychology of students and it has provided a lot of highquality training materials and video lessons for the majority of learners. Then, the teaching contents will be much richer, the teaching will be more skilled and the teaching effect will be more prominent. At the same time, the students can easily understand the arcane psychological knowledge through watching the loved animation works. Afterwards, according to their actual situations , they canre-examine their own inner world, which the students obtain the full development in the aspects of self-awareness, self-reliance, adaption of environment and selection of careers, etc. Meanwhile, the psychological quality and the personality of college students can be also improved.

\section{B. Module of interesting psychology}

In addition to the general artistic characteristics, the comic also has its own features, which can help it play the function of psychotherapy. Firstly, the comic is a popular art. It closely contacts with the real life, which can more sensitive, more extensive and more detailed to reflect the characteristics of times. Secondly, the humor is the most important feature of comics and this feature itself has the therapeutic implications. Therefore, the psychotherapy significance of comics is an inevitable trend. In this module, we can collect or draw a lot of psychological comics and the cartoons can be adopted to reflect the deep thinking for life. At the same time, some life wisdoms can be also explained through adopting the unique comic language combining with the psychological knowledge.

\section{Module of interesting test}

According to the psychological characteristics of students, this module prepares some interesting self-test questions for the students. These tests can be presented by the flash 2D animations. The common text test can be transformed to the interactive game program test through adopting the interactive design functions of flash animation, which makes the test process be more interesting and more situational. At the same time, the students will keen on self-understanding and self-cognition. According to the different test results, some relevant suggestions will be provided to optimize the psychological quality of students, to prevent and mitigate the may arising psychological problems.

\section{Module of case study}

It is required to select one or a few students as the research subjects in this module. And it is also necessary to record the mental performance or the psychological problems of students to form the cases. Afterwards, the production staff will adapt the cases into the scripts and they will be developed into 2D or 3D animation short films. In these short films, the comprehensive analysis for each case can be conducted according to the recommendations of the one hand, according to the contents of free browsing cases and the targeted suggestions proposed by teachers the mental health teachers. And some advice or treatment suggestions can be also provided. On, the students will learn the selfobservation and self-management. On the other hand, it is required to introduce the cases into the learning resources with the form of animation, which can be helpful to protect the privacy of students, respect for the dignity of students and prevent the secondary psychological harm.

\section{E. Module of psychological theater}

Generally speaking, the psychological drama contains six basic elements: director, protagonist, stage, substitute, auxiliary role and audience. If each psychological drama can be presented by the real stage performance form, a lot of manpower and resources will be required. In order to solve the above problems and initiate the catharsis in the process of watching psychological dramas, this module transforms the true stage performance form into the low-cost animation short films which can be easily developed. Then, the students will have the feelings to purify the grief, the sadness and the pressure in their lives due to the flow of emotions in the process of watching, which can cultivate the sentiments of students and build up the positive outlook on life, the world outlook and the moral values.

\section{F. Module of works show}

It is required to conduct the display and exchange activities through this module so as to guide the teachers and the students to conduct the "Comics and Psychology" and "Animation and Psychology" works show in the platform as well as expand the influence range of animation resources in the psychological health education. According to the file submission functions provided by system, the name can be freely given and the self-made animation works can be released. On the one hand, the animation works about the psychological health education can be applied in the mental health education of college students in campus. On the other hand, in the display process, the teachers can also participate in the production of resources, which can improve the research ability of teachers. At the same time, the students can be also allowed to join in the creation of mental health education stories and the production of animations, which can improve their mental knowledge literacy as well as the media literacy.

The method that combines the production of animation resources with the mental health education of college students has broken through the previous knowledge that the animation only has the entertainment features. The construction of the new educational resources and 
educational methods through exploring and developing the educational functions and values of animation is of great value in the promotion of the development of current education reform and the effective improvement of students' psychological health education.

\section{ACKNOWLEDGMENT}

This study was supported by Education and Teaching Research Project of Suzhou Vocational University (SZDJG12006)

\section{REFERENCES}

[1] Liu Cang-hai, Zhong Ping. Analysis on China s Animation Resources-Blue Cat and China s Animation Industry Development. Journal of Jiujiang Vocational and Technical College, 2010,10(02), pp.91-92.
[2] YAO Gui-ping The Exploitation and Using of Curriculum Resource of Mental Health Education in Vocational Schools. Vocational and Technical Education,2003.24(10),61-63.

[3] Allan, R.Walt Disney and Europe-European Influences on the Animated Feature Films of Walt Disney, John Libbey,1998.

[4] Barrier,M. Hollywood Cartoons: American Animation in Its Golden Age, New York: Oxford University Press,1999.

[5] Zhang Xiangkui.Self-respect: An Important Psychological Resource for School Education. Educational Research, 2003,03(01),pp.5457,2003 .

[6] HUANG Xue-xiong.Exploitation and Utilization of Psychology Resources in University Libraries under Scientific Concept of Development. Journal of Library and Information Sciences in Agriculture,2010,22(06),163-166. 